\title{
Singular separatrix splitting and the Poincaré-Melnikov method for area preserving maps
}

\author{
Amadeu Delshams and Rafael Ramírez-Ros \\ Departament de Matemàtica Aplicada I \\ Universitat Politècnica de Catalunya \\ Diagonal 647, 08028 Barcelona, Spain
}

\begin{abstract}
The splitting of separatrices of area preserving maps close to the identity is one of the most paradigmatic examples of an exponentially small or singular phenomenon. The intrinsic small parameter is the characteristic exponent $h>0$ of the saddle fixed point. A standard technique to measure the splitting of separatrices is the so-called Poincaré-Melnikov method, which has several specific features in the case of analytic planar maps. The aim of this talk is to compare the predictions for the splitting of separatrices provided by the Poincaré-Melnikov method, with the analytic and numerical results in a simple example where computations in multiple-precision arithmetic are performed.
\end{abstract}

\section{Introduction}

In this talk, we review some results concerning the singular splitting of separatrices for the family of planar standard-like maps

$$
F(x, y)=\left(y,-x+U^{\prime}(y)\right)=\left(y,-x+\frac{2 \mu_{0} y}{1+y^{2}}+\varepsilon V^{\prime}(y)\right),
$$

where $V(y)=\sum_{n \geq 1} V_{n} y^{2 n}$ is an even entire function. These maps are area preserving.

If $\mu_{0}+V_{1} \varepsilon>1$, the origin $O=(0,0)$ is a saddle point with $\operatorname{Spec}\left[D F_{0}(O)\right]=\left\{\mathrm{e}^{h}, \mathrm{e}^{-h}\right\}$, where the characteristic exponent $h>0$ is given by $\cosh h=\mu_{0}+V_{1} \varepsilon$. In the weakly hyperbolic situation $\left(h \rightarrow 0^{+}\right)$, the splitting size turns out to be exponentially small in $h$, so that its measure requires a very careful treatment, both from a numerical and an analytical point of view.

Our choice of the above maps, instead of more celebrated standard-like maps like the Hénon map or the standard map, is due to the fact that some explicit exponentially small estimates of the splitting size can be obtained for those maps. The estimates are computed using a discrete version of the Poincaré-Melnikov method.

This talk is organized as follows. In section 2, the discrete version of the Poincaré-Melnikov method for twist maps is described. Section 3 contains the analytical results about the splitting, both in the regular (Theorem 1) and singular (Theorem 2) cases. Finally, the numerical experiments are presented in section 4 .

\section{The Poincaré-Melnikov method}

Let $F_{0}: \mathbb{R}^{2} \rightarrow \mathbb{R}^{2}$ be a twist diffeomorphism with twist generating function $\mathcal{L}_{0}$, that is, $(X, Y)=$ $F_{0}(x, y)$ if and only if $y=-\partial_{1} \mathcal{L}_{0}(x, X)$ and $Y=\partial_{2} \mathcal{L}_{0}(x, X)$. We will assume that $F_{0}$ has a separatrix $\Lambda$ to a saddle point $z_{0}^{\infty}=\left(x_{0}^{\infty}, y_{0}^{\infty}\right) \notin \Lambda$; that is, the invariant curves of $z_{0}^{\infty}$ coincide along the separatrix $\Lambda$.

Let $F: \mathbb{R}^{2} \rightarrow \mathbb{R}^{2}$ be a twist perturbation of $F_{0}$, with generating function $\mathcal{L}=\mathcal{L}_{0}+\varepsilon \mathcal{L}_{1}+\mathrm{O}(\varepsilon)$. For $|\varepsilon|$ small enough, $F$ has a saddle point $z^{\infty}$ "close" to $z_{0}^{\infty}$, whose local invariant curves are 
"close" to the unperturbed separatrix, although, in general, the perturbed invariant curves no longer coincide.

To compute their distance, it is standard to invoke the Poincaré-Melnikov method which, in the case of area preserving maps, is based on the properties of the Melnikov potential (see [2, 6] and references therein)

$$
L(z)=\sum_{k \in \mathbb{Z}}\left[\mathcal{L}_{1}\left(x_{k}, x_{k+1}\right)-\mathcal{L}_{1}\left(x_{0}^{\infty}, x_{0}^{\infty}\right)\right], \quad z_{k}=\left(x_{k}, y_{k}\right)=F_{0}^{k}(z), \quad z \in \Lambda .
$$

If $L$ has a non-degenerate critical point at $z=z_{0}$, then there exists a transverse homoclinic point $z_{\mathrm{h}}=z_{0}+\mathrm{O}(\varepsilon)$ of the perturbed map. Moreover, if $z_{0}$ and $z_{0}^{\prime}$ denote consecutive non-degenerate critical points of $L$, the pieces of the perturbed invariant curves between the associated homoclinic points $z_{\mathrm{h}}$ and $z_{\mathrm{h}}^{\prime}$ enclose a region called lobe, whose area is invariant under $F$. This lobe area $A$ has a nice expression in terms of the Melnikov potential:

$$
A=\varepsilon\left[L\left(z_{0}^{\prime}\right)-L\left(z_{0}\right)\right]+\mathrm{O}\left(\varepsilon^{2}\right) .
$$

There exist similar theories for $2 d$-dimensional exact symplectic maps and Hamiltonian flows (see $[3,1]$ and references therein).

\section{Analytical results}

We are now ready to apply the Poincaré-Melnikov method to our maps.

To begin with, the maps (1) are twist maps with generating function $\mathcal{L}(x, X)=-x X+U(X)$. Moreover, the origin is a saddle point with characteristic exponent $h>0, \cosh h=\mu$, provided that $\mu:=\mu_{0}+V_{1} \varepsilon>1$.

It is convenient to split the function $U(y)=\mu_{0} \log \left(1+y^{2}\right)+\varepsilon V(y)$ as $U(y)=U_{0}(y)+\varepsilon U_{1}(y)$, with $U_{0}(y)=\mu \log \left(1+y^{2}\right)$ and $U_{1}(y)=V(y)-V_{1} \log \left(1+y^{2}\right)$. Then the maps (1) take the form

$$
F(x, y)=F_{0}(x, y)+\varepsilon F_{1}(x, y), \quad F_{0}(x, y)=\left(y,-x+U_{0}(y)\right), \quad F_{1}(x, y)=\left(0, U_{1}(y)\right),
$$

and so $\mathcal{L}(x, X)=\mathcal{L}_{0}(x, X)+\varepsilon \mathcal{L}_{1}(x, X)$, with $\mathcal{L}_{0}(x, X)=-x X+U_{0}(X)$ and $\mathcal{L}_{1}(X)=U_{1}(X)$.

When $\varepsilon=0$, the unperturbed map

$$
F_{0}(x, y)=\left(y,-x+U_{0}^{\prime}(y)\right)=\left(y,-x+\frac{2 \mu y}{1+y^{2}}\right)
$$

is called McMillan map [8] and is integrable, with a polynomial first integral given by

$$
I_{0}(x, y)=x^{2}-2 \mu x y+y^{2}+x^{2} y^{2} .
$$

Since $\mu>1$, the zero level of $I_{0}$ is a lemniscate, whose loops are separatrices to the origin. The separatrix $\Lambda$ in the quadrant $\{x, y>0\}$ can be parameterized by

$$
z_{0}(t)=\left(x_{0}(t), y_{0}(t)\right)=\left(\xi_{0}(t-h / 2), \xi_{0}(t+h / 2)\right), \quad \xi_{0}(t)=\gamma \operatorname{sech} t, \quad \gamma=\sinh h .
$$

which is a natural parameterization; that is, $F_{0}\left(z_{0}(t)\right)=z_{0}(t+h)$.

When $\varepsilon \neq 0$, the map $F$ can be thought as a perturbed McMillan map, although the characteristic exponent of the origin does not change, since the perturbation $\varepsilon U_{1}^{\prime}(y)$ begins with cubic terms. These two parameters, the characteristic exponent $h>0$ and the perturbation strength $\varepsilon$, will be considered the intrinsic parameters of our family of maps. 
Since $U(y)$ is an even function, $F$ is an odd map, and thus the perturbed invariant curves of the origin are symmetric with respect to the origin, so that we concentrate only on the positive quadrant $\{x, y>0\}$.

By the form of the perturbation, $R^{+}(x, y)=(y, x)$ is a reversor of $F$, as well as the involution $R^{-}:=F \circ R^{+}$, which is given by $R^{-}(x, y)=\left(x,-y+U^{\prime}(x)\right): F_{0}^{-1}=R^{ \pm} \circ F_{0} \circ R^{ \pm}$. Consequently, the perturbed invariant curves intersect at points $z^{ \pm} \in C^{ \pm}=\left\{z: R^{ \pm} z=z\right\}$, which give rise to two primary symmetric homoclinic orbits $\mathcal{O}^{ \pm}$on the quadrant $\{x, y>0\}$.

Using the parameterization (4), the Melnikov potential (2) of our problem is given by

$$
L(t):=L\left(z_{0}(t)\right)=\sum_{n \in \mathbb{Z}} U_{1}\left(y_{0}(t+h n)\right)=\sum_{n \in \mathbb{Z}} V\left(\xi_{0}(t+h / 2+h n)\right),
$$

and is a doubly-periodic function: $L(t)=L(t+h)=L(t+\pi \mathrm{i})$. As a consequence, the series of $L(t)$ can be computed explicitly through the study of the complex singularities of $L(t)$ [2], leading to the expression (modulo an additive constant):

$$
L(t)=\mathrm{e}^{-\pi^{2} / h} \cos (2 \pi t / h)\left[-\Theta^{0}(h) / 2+\mathrm{O}\left(\mathrm{e}^{-2 \pi^{2} / h}\right)\right],
$$

in terms of an even entire function $\Theta^{0}(h)$ that satisfies

$$
\Theta^{0}(h)=8 \pi \sum_{n \geq 1} \frac{(2 \pi)^{2 n-1}}{(2 n-1) !} V_{n}+\mathrm{O}\left(h^{2}\right)=8 \pi \widehat{V}(2 \pi)+\mathrm{O}\left(h^{2}\right),
$$

where $\widehat{V}(\xi):=\sum_{n \geq 1} V_{n} \xi^{2 n-1} /(2 n-1)$ ! is the so-called Borel transform of $V(y)$. We refer to [4] for the details.

From formula (5), one sees that the Melnikov potential $L(t)$ is $\mathrm{O}\left(\exp \left(-\pi^{2} / h\right)\right)$, and that for $\widehat{V}(2 \pi) \neq 0$ and $h$ small enough, all the critical points of $L(t)$ are non-degenerate and contained in $h \mathbb{Z} / 2$. Therefore, the Poincaré-Melnikov formula (3) gives the following result.

Theorem 1 Assume that $\widehat{V}(2 \pi) \neq 0$. Then, for $0<|\varepsilon|<\varepsilon^{*}(h)=\mathrm{o}\left(\exp \left(-\pi^{2} / h\right)\right)$, the map $(1)$ has exactly two transverse, symmetric, primary homoclinic orbits in the quadrant $\{x, y>0\}$. These orbits determine a lobe with area $A=\varepsilon A_{\mathrm{Mel}}+\mathrm{O}\left(\varepsilon^{2}\right)$, where the first order in $\varepsilon$ approximation $A_{\mathrm{Mel}}$ is given by

$$
A_{\mathrm{Mel}}=L(h / 2)-L(0)=\mathrm{e}^{-\pi^{2} / h}\left[\Theta^{0}(h)+\mathrm{O}\left(\mathrm{e}^{-2 \pi^{2} / h}\right)\right] .
$$

In the singular case $\left(h \rightarrow 0^{+}\right)$and $\varepsilon=\mathrm{O}\left(h^{p}\right), p>0$, Theorem 1 cannot be applied, since it requires $\varepsilon$ to be exponentially small in $h$. For $p>6$, the following theorem [4, 5] gives an asymptotic expression for the lobe area in terms of the Melnikov potential, and establishes transversal splitting of separatrices.

Theorem 2 Assume that $\varepsilon=\mathrm{o}\left(h^{p}\right), p>6$. Then, if $\widehat{V}(2 \pi) \neq 0$, there exists $h^{*}>0$ such that the map (1) has exactly two transverse, symmetric, primary homoclinic orbits in the first quadrant, for all $0<h<h^{*}$. Moreover, they enclose a lobe with area

$$
A=\varepsilon \mathrm{e}^{-\pi^{2} / h}\left[8 \pi \widehat{V}(2 \pi)+\mathrm{O}\left(h^{2}\right)\right] \quad\left(h \rightarrow 0^{+}\right) .
$$


As a consequence, for $\varepsilon=\mathrm{o}\left(h^{p}\right), p>6$, the lobe area $A$ is given, in first order, by the PoincaréMelnikov method. At this very moment, one is confronted with several questions about the expression of the lobe area $A=A(h, \varepsilon)$ in the singular case $h \rightarrow 0^{+}$:

1. Is the lobe area $A$ also given by the Poincaré-Melnikov for $\varepsilon=\mathrm{o}\left(h^{p}\right), 0<p \leq 6$ ?

2. Is $A$ also given by the Poincaré-Melnikov for $\varepsilon \rightarrow 0$ independently of $h \rightarrow 0^{+}$?

3. What is the expression for $A$ for $\varepsilon$ fixed and $h \rightarrow 0^{+}$?

The singular cases 1 and 2 are perturbative, whereas the singular case 3 is non-perturbative. To deal with them, we performed some numerical experiments to compute the lobe area $A$, as explained in the next section.

\section{Numerical results}

We now study numerically the cases not covered by the analytical results in the singular limit.

The computation of the lobe area $A$ near the singular limit is a delicate question, since several numerical problems are intrinsic to it. To mention only a few: (i) the dynamics near the saddle point is extremely slow, due to the weakly hyperbolic character of the fixed point; (ii) the lobe area is computed as a difference of exponentially close actions, giving rise to a massive cancellation of significant digits; and (iii) the general algorithms for computing homoclinic points have condition numbers inversely proportional to the splitting size, being exponentially illconditioned for our singular maps.

To overcome these difficulties, we are forced to: (i') expand the local invariant curves up to very high (but optimal) order; (ii') use an expensive multiple-precision arithmetic; and (iii') take the greatest advantage of symmetries and/or reversors. For instance, the computations in the most extreme cases (in which $h=0.001$ and the lobe area $A$ was less than $10^{-4200}$ ) have required more than 5200 digits in the arithmetic expanding the invariant curves up to order 1300 . We refer to [5] for the details and the implementation of the programs.

All these items encourage us to restrict the numerical experiments to the simplest perturbations; that is, for the linear perturbation $\varepsilon V^{\prime}(y)=\varepsilon y$, and the cubic one $\varepsilon V^{\prime}(y)=\varepsilon y^{3}$.

When $V(y)$ is a polynomial the Melnikov potential $L(t)$ is an elliptic function, and $\Theta^{0}(h)$ can be explicitly computed in a finite number of steps [2]:

$$
\Theta^{0}(h)=\left\{\begin{array}{ll}
8 \pi^{2} \gamma^{2} h^{-2} & \text { for } V^{\prime}(y)=y \\
\frac{8}{3} \pi^{2} \gamma^{4} h^{-2}\left[1+\pi^{2} h^{-2}\right] & \text { for } V^{\prime}(y)=y^{3}
\end{array} .\right.
$$

Taking into account the expression (7) of Theorem 1, in the singular case we look, for every fixed $\varepsilon$, for a formula for the lobe area of the form

$$
A=\varepsilon \mathrm{e}^{-\pi^{2} / h}\left[\Theta^{\varepsilon}(h)+\mathrm{O}\left(\mathrm{e}^{-2 \pi^{2} / h}\right)\right], \quad\left(\text { fixed } \varepsilon, h \rightarrow 0^{+}\right),
$$

for a function $\Theta^{\varepsilon}(h)$ given by an asymptotic series of the form

$$
\Theta^{\varepsilon}(h) \sim \sum_{n \geq 0} \Theta_{n}^{\varepsilon} h^{2 n}, \quad\left(\text { fixed } \varepsilon, h \rightarrow 0^{+}\right) .
$$


The sign $\sim$ means that the series above needs not to be convergent, but only asymptotic: for every $N \geq 0$,

$$
\left|\Theta^{\varepsilon}(h)-\sum_{n=0}^{N} \Theta_{n}^{\varepsilon} h^{2 n}\right|=\mathrm{O}\left(h^{2 N+2}\right) .
$$

After computing and analyzing a relevant number of coefficients $\Theta_{n}^{\varepsilon}$ (up to $n=100$, say), the numerical results obtained for $\varepsilon$ fixed and $h \rightarrow 0^{+}$(the non-perturbative case) can be summarized as follows.

Numerical result 1 For the linear and cubic perturbations, the following asymptotic expansion for the lobe area $A$ holds

$$
A \sim \varepsilon \mathrm{e}^{-\pi^{2} / h} \sum_{n \geq 0} \Theta_{n}^{\varepsilon} h^{2 n} \quad\left(\varepsilon \text { fixed }, h \rightarrow 0^{+}\right)
$$

where the coefficients $\Theta_{n}^{\varepsilon}$ verify

$$
\Theta_{n}^{\varepsilon}=(2 n) !\left(2 \pi^{2}\right)^{-2 n}(2 n)^{4}\left[\Xi_{\infty}^{\varepsilon}+\mathrm{O}\left(n^{-1}\right)\right]
$$

as $n \rightarrow+\infty$, for some constant $\Xi_{\infty}^{\varepsilon} \neq 0 .\left(\Xi_{\infty}^{\varepsilon}<0\right.$ for $\varepsilon>0$.)

In particular, the series $\Theta^{\varepsilon}(h)=\sum_{n>0} \Theta_{n}^{\varepsilon} h^{2 n}$ is divergent but it is Gevrey-1, i.e., its Borel transform $\widehat{\Theta^{\varepsilon}}(h)=\sum_{n} \Theta_{n}^{\varepsilon} \xi^{2 n-1} /(2 n-1)$ ! is convergent, for $|\xi|<2 \pi^{2}$.

The next step is to consider the perturbative case, and therefore to check whether all the previous objects $\Theta^{\varepsilon}(h), \Theta_{n}^{\varepsilon}, \Xi_{\infty}^{\varepsilon}$, tend to well-defined limits, as $\varepsilon \rightarrow 0$. This was confirmed by the numerical experiments.

Numerical result 2 For the linear and cubic perturbations, the objects $\Theta^{\varepsilon}(h), \Theta_{n}^{\varepsilon}, \Xi_{\infty}^{\varepsilon}$, introduced in the Numerical Result 1, tend to well-defined limits, as $\varepsilon \rightarrow 0$. More precisely,

1. $\Theta^{\varepsilon}(h)=\Theta^{0}(h)+\mathrm{O}(\varepsilon)$, uniformly in $h \in(0,1]$.

2. $\Theta_{n}^{\varepsilon}=\Theta_{n}^{0}+\mathrm{O}(\varepsilon)$, non-uniformly in $n \geq 0$.

3. $\Xi_{\infty}^{\varepsilon}=\varepsilon \Xi_{\infty}^{0}+\mathrm{O}\left(\varepsilon^{2}\right)$, where $\Xi_{\infty}^{0}=\left\{\begin{array}{ll}-12 \pi^{-4} & \text { if } V^{\prime}(y)=y \\ -16 / 3 & \text { if } V^{\prime}(y)=y^{3}\end{array}\right.$.

\section{Closing}

From the numerical results computed for the map (1), one sees that the Melnikov potential provides the right answer even in the singular case $\varepsilon \rightarrow 0$ independently of $h \rightarrow 0^{+}$. Therefore, one should improve analytically the hypothesis $\varepsilon=\mathrm{o}\left(h^{p}\right), p>6$ in Theorem 2 .

As a matter of fact, the Numerical result 1 suggests that resurgence tools [7] may be successful to fill this gap between analytical and numerical results. Asymptotic expressions similar to (11) seem to be true [9] for some celebrated entire maps, like the Hénon map or the standard map.

\section{Acknowledgments}

This work was partially supported by the Catalan grant CIRIT 1998SGR-00041. Research by A. Delshams is also supported by the Spanish grant DGICYT PB94-0215. 


\section{References}

[1] A. Delshams and P. Gutiérrez. Splitting potential and Poincaré-Melnikov theory for whiskered tori in Hamiltonian systems. Preprint, 1999. 2

[2] A. Delshams and R. Ramírez-Ros. Poincaré-Melnikov-Arnold method for analytic planar maps. Nonlinearity, 9(1):1-26, 1996. 2, 3, 4

[3] A. Delshams and R. Ramírez-Ros. Melnikov potential for exact symplectic maps. Comm. Math. Phys., 190:213-245, 1997. 2

[4] A. Delshams and R. Ramírez-Ros. Exponentially small splitting of separatrices for perturbed integrable standard-like maps. J. Nonlinear Sci., 8(3):317-352, 1998. 3, 3

[5] A. Delshams and R. Ramírez-Ros. Singular separatrix splitting and the Melnikov method: an experimental study. Experiment. Math., 8(1):29-48, 1999. 3, 4

[6] A. Delshams, R. Ramírez-Ros, and T.M. Seara. Splitting of separatrices in Hamiltonian systems and symplectic maps. In C. Simó, editor, Proceedings of the NATO Advanced Study Institute held in s'Agaró, Spain, June 19-30, 1995, volume 533 of NATO ASI Ser. C: Math. Phys. Sci., pages 39-54, Dordrecht, Holland, 1999. Kluwer Acad. Publ. 2

[7] V. Gelfreich and D. Sauzin. Exponentially small splitting of separatrices near bifurcations in areapreserving maps. These proceedings, 1999. 5

[8] E.M. McMillan. A problem in the stability of periodic systems. In E. Brittin and H. Odabasi, editors, Topics in modern physics, a tribute to E.V. Condon, pages 219-244. Colorado Assoc. Univ. Press, Boulder, CO, 1971. 3

[9] C. Simó. Analytical and numerical detection of exponentially small phenomena. These proceedings, 1999. 5

\section{Internet access:}

All the authors' quoted preprints, as well as the code for the programs used in this paper, are available at http://www-ma1.upc.es in the Preprints pages, or at ftp://ftp-ma1.upc.es, in the pub/preprints directory.

E-mail addresses of the authors: amadeu@ma1.upc.es, rafael@tere.upc.es. 